\title{
Attention!!! Is 2 weeks cough symptoms is enough or still 3 weeks cough symptoms can assess tuberculosis
}

\author{
Sumeet David ${ }^{1 *}$, Navjot Singh $^{2}$, Aroma Oberoi ${ }^{3}$, Ashish Chawla ${ }^{4}$ \\ ${ }^{1}$ Department of medicine, Christian medical college \& hospital, Ludhiana, Punjab \\ ${ }^{2}$ department of medicine, Christian medical college \& hospital, Ludhiana, Punjab \\ ${ }^{3} \mathrm{HOD}$, department of microbiology, Christian medical college \& hospital, Ludhiana, Punjab \\ ${ }^{4}$ TB Officer, Department of Chest Medicine, Civil Hospital, Ludhiana, Punjab \\ *Corresponding author E-mail: sumeetdavid_2007@yahoo.co.in
}

Copyright () 2014 Dr. Sumeet David.et.al. This is an open access article distributed under the Creative Commons Attribution License, which permits unrestricted use, distribution, and reproduction in any medium, provided the original work is properly cited.

\begin{abstract}
A prospective cross sectional study was conducted in the Department of Medicine, Christian Medical College and Hospital and Civil Hospital, Ludhiana. Patients between the age group 18-70 years were included in the study. 790 patients were taken for the study. Numbers of males were $65.4 \%$ and females $34.6 \%$. In the study, it was found that in patients with 2 weeks cough duration, sputum AFB was positive at 2 weeks as well as 3 weeks. But in those patients who were negative at 2 weeks duration, when they were re-examined at 3rd week, the positivity of sputum AFB had increased which was statistically significant. So sputum analysis at 3 weeks is better than 2 weeks for tuberculosis evaluation, although evaluation of sputum should be done early at 2 weeks, cough duration for early diagnosis and treatment initiation.
\end{abstract}

Keywords: smear positive tuberculosis, chest symptoms, cough duration.

\section{Introduction}

The present study is a dots controlled study. In this study cough symptomatic at 2 weeks were assessed. Positive samples at 2 weeks which were repeated after 2 weeks came positive. Out of which who came negative at 2 weeks positivity was increased when they were repeated again at 2 weeks. This study is seen in same group of patients but the studies which are done in 2005 and 2007 are in different group of patients. In 2005 and 2007 multicenteric study was done in out-patients found that detection of smear Positive tuberculosis can be substantially improved by actively eliciting history and by changing The screening criterion for performing sputum microscopy among out-patients from cough $\geq 3$ Weeks to $\geq 2$ weeks 4,13 .

More than five million cases of tuberculosis were reported to the World Health Organization (WHO) in 2005. More than $90 \%$ were reported from developing countries.

Targets were established to measure the progress of implementation of the directly observed treatment, short - course (DOTS) strategy to combat tuberculosis by 2005 . These targets are $100 \%$ population Coverage with DOTS, $70 \%$ case detection and $85 \%$ treatment success rate. Achieving these Targets is an important intermediate step towards reaching the impact targets of 50\% reduction in prevalence and mortality due to TB by 2010 as compared to 2000 targets 4 .

In 1992, Government of India together with the WHO and Swedish International development agency (SIDA), reviewed the national TB programme and concluded that it suffered from managerial weakness, inadequate funding, over-reliance on x-ray, non-standard treatment, regimens, low rates of treatment completion, and lack of systemic information on treatment outcomes (RNTCP 2007 Report).

Coverage for DOTS services, at least in the public primary care network has reached nearly $100 \%$ in late 2002. Ever since, diagnosis through sputum smear microscopy and treatment with a complete set of anti-TB drugs are given free through the support of the Government (2010-2016 Philippine Plan of Action to Control TB and DOTS report). 


\section{Material and methods}

The study was a prospective cross sectional study conducted from 1st August 2008 to 31st July, 2009 in the Department of Medicine, Christian Medical College and Hospital and Civil Hospital Ludhiana. According to a quarterly report in 2009. Ludhiana is known to be endemic in Tuberculosis with 159 TB suspects per lakhs population, 63\% annualized new sputum positive case detection rate, sputum conversion rate new cases is $92 \%$.

790 patients between the age group of 18-70 years were included in this study. Patients with 2 weeks cough duration, sputum AFB was positive at 2 weeks as well as 3 weeks and those patients who were negative at 2 weeks duration, when they were re-examined at 3rd week, positivity was increased. Numbers of males examined were $65.4 \%$ and numbers of females were $34.6 \%$. Out of the patients observed only $14 \%$ were uneducated and laborers. Rest all were educated and servicemen. This shows that tuberculosis nowadays is seen in effluent societies of India. Verbal consent was taken from the participants and written permission to conduct the study was taken from ethics committee and review board of CMC \& H Ludhiana and Dr Ashish Chawla (TB officer), Civil Hospital Ludhiana, Punjab.

Sputum smear examination at a peripheral centre is done by Z-N staining (Ziehl-Neelsen). Components of Z-N staining

1) Primary stain: strong carbol fuchsin.

2) Decolourizer: $20 \%$ sulphuric acid or acid alcohol.

3) Counterstain: Loeffler's methylene blue or malachite green.

\section{Procedure of Z-N staining}

The sputum specimen is smeared uniformly in a small area over a new, scratch-free glass slide and fixed. The slide is placed on a staining rack and is flooded by strong carbol fuchsin solution. The slide is intermittently heated (for 5 minutes) from underneath using a spirit lamp until fumes arise. Care must be taken not to boil the solution or drying of the slide. The slide should be washed in water and decolorized by $20 \% \mathrm{H} 2 \mathrm{SO} 4$ until the slide is almost colorless or pale pink. Decolourization generally requires contact with sulphuric acid for a total time of at least 10 minutes. The smear is then washed and counterstained with Loeffler's blue solution for 1-2 minutes. The slide is washed again and dried.

\section{Inclusion criteria}

Patients who presented with two weeks cough are included in the study.

\section{Exclusion criteria}

Patients who are already on ATT.

\section{Result}

The present study which is conducted in 790 patients is done under same group of patients but in previous two studies comparison was done in different group of patients. Patients with cough symptoms of two weeks duration underwent sputum examination for AFB. This is a totally new approach of assessing same group of patients who comes at 2 weeks followed by 3 weeks:

1) The objective is to assess the sputum positivity in 2 weeks versus 3 weeks chest symptomatology in Pulmonary Tuberculosis and Use of 2 versus 3 sputum specimens for the diagnosis of Pulmonary Tuberculosis.

2) In table 1, a total of 790 patients underwent sputum AFB examination. 1st sputum sample at 2 weeks was positive came in 149 patients out of total 790 patients. The analysis of the data showed that the 1 st specimen (spot) could detect $18.9 \%(149 / 790)$ of all patients in same group

3) In table 2, a total of 790 patients underwent sputum AFB examination. 2nd sputum sample at 2 weeks was positive came in 149 patients out of total 790 patients. The analysis of the data showed that the 2 nd specimen (spot) could detect $18.9 \%(149 / 790)$ of all patients in same group.

4) In table 3, a total of 790 patients underwent sputum AFB examination. 3rd sputum sample at 2 weeks was positive in 150 patients out of total 790 patients. The analysis of the data showed that the 3rd specimen (spot) could detect $19.0 \%(150 / 790)$ of all patients in same group.

5) In table 4, out of the total 790 patients who came sputum positive at 2 weeks are positive at 3 weeks also i.e. 149 in 2 weeks and 148 in 3 weeks respectively. Out of which 641 which were negative at 2 weeks when they were followed up at 3 weeks 6 came positive which was statistically significant $(\mathrm{p}<0.00)$. One case turned out to be false negative. This study was done in same group of patients. So, there is $0.9 \%$ increase in the smear positive in the 3 rd week and total percentage in 3rd week is (154/790) 19.5\% increase. As compared in Santha et al (2005) positivity has increased of $61 \%$ of chest symptomatic and $46 \%$ increased detected in smear positive cases in two weeks and in Thomas et al (2008) there is overall increase of 58\% increase in chest symptomatic and $23 \%$ in smear positive cases these studies done in different group of patients. 


\section{Discussion}

In present study, it was found that in patients with two weeks cough history, sputum AFB was positive at two weeks as well as three weeks. But in those patients who are negative at two weeks duration, when they were re-examined at three weeks duration the positivity for sputum AFB has increased which was statistically significant. So sputum analysis at three weeks is better than two weeks for Tuberculosis evaluation, although evaluation of sputum should be done early at two weeks, cough duration for early diagnosis and treatment initiation.

Table 1: 1st sputum sample in two weeks

\begin{tabular}{ccc}
\hline Sputum samples & Frequency & Percentage \\
\hline Negative & 641 & 81.1 \\
Positive & 149 & 18.9 \\
Total & 790 & 100.0 \\
\hline
\end{tabular}

Table 1 depicted that out of 790 patients 1 st sputum sample in the two weeks show a positivity of $149(18.9 \%)$ and negativity of $641(81.1 \%)$ patients.

\begin{tabular}{ccc} 
& Table 2: 2nd Sputum Sample in Two Weeks & Percentage \\
\hline Sputum samples & Number of patients & 81.1 \\
Negative & 641 & 18.9 \\
Positive & 149 & 100.0 \\
Total & 790 & \\
\hline
\end{tabular}

Table 2 and Figure 2 depicted that out of 790 patients 1st sputum sample in the two weeks show a positivity of 149 $(18.9 \%$ and negativity of $641(81.1 \%)$ patients.

Table 3: 3rd Sputum Sample in Two Weeks

\begin{tabular}{ccc}
\hline Sputum samples & Number of patients & Percentage \\
\hline Negative & 640 & 81.0 \\
Positive & 150 & 19.0 \\
Total & 790 & 100.0 \\
\hline
\end{tabular}

Table 3 depicted that out of 790 patients 1st sputum sample in the two weeks show a positivity of $150(19.0 \%)$ and negativity of $640(81.0 \%)$ patients.

Table 4: 1st Sputum sample in two weeks cough symptomatology Vs three weeks cough Symptomatology

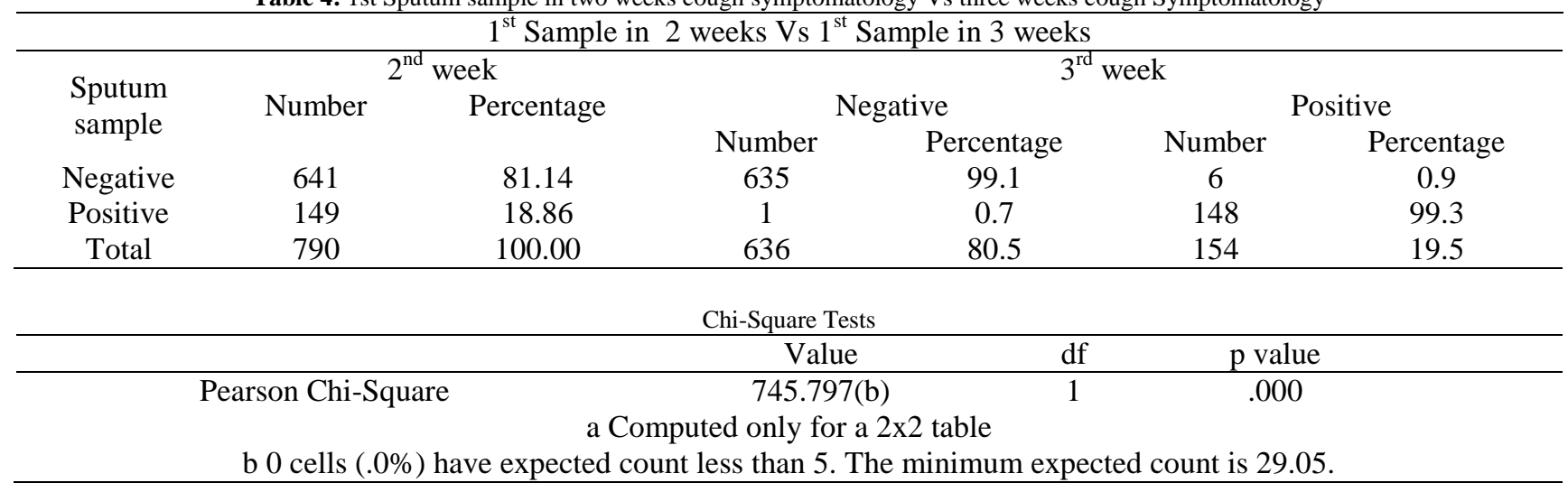

This Table 4 shows 1 st sputum sample at 2 weeks cough symptomatology shows positivity of 149 , when these patients were followed up at 3 weeks positivity remains the same. Out of 641 which were negative and which were followed up at 3 weeks 6 came positive which is statistically significant ( $\mathrm{p} 0.000)$.

\section{Acknowledgements}

We are grateful to the doctors, laboratory technologists and other staff of the DOTS Centre of Christian Medical College \& Hospital Ludhiana and Civil Hospital Ludhiana for or their enormous cooperation and assistance in collecting samples for this study. 


\section{References}

[1] Raviglione MC, O’Brien RJ,Anthony S, Fauci, Dennis L, Kasper, Dan L. Longo, Eugene Braunwald, Stephen L. Hauser, J. Larry Jaeson (2008). Mycobacterial Diseases - Tuberculosis: Harrison's Principle of Medicine, McGraw Hill, New York, Volume 1, 17th Edition, Chapter 158 (Eds.), 1006-1020.

[2] Gopi PG, Subramani R, Santha T, Chandrasekaran V, Kolapan C, Selvakumar N, et al(2005) : Estimation of burden of tuberculosis in India for the year 2000. Indian J Med Res; 122: 243-248.

[3] Chadha VK: Tuberculosis epidemiology in India: a review (2005) . Int J Tuberc Lung Dis; 9 (10): 1072-1082.

[4] Santha T, Garg R, Subramani R, Chandrasekaran V, Selvakumar N. et al (2005): Comparison of cough of 2 and 3 weeks to improve detection of smear-positive tuberculosis cases among out-patients in India. Int J Tuberc Lung Dis; 9 (1): 61-68.

[5] Golub JE, Mohan CI, Comstock GW, Chaisson RE (2005). Active case finding of tuberculosis: historical perspective and future prospects. Int J Tuberc Lung Dis; 9 (11): 1183-1203.

[6] English RG, Bachmann MO, Bateman ED, Zwarenstein MF, Fairall SR, Bheekie A, et al (2006). Diagnostic accuracy of an integrated respiratory guideline in identifying patients with respiratory symptoms requiring screening for pulmonary tuberculosis: a cross-sectional study. BMC Pulm Med; 6: 22.

[7] Harris AD, Mphasa NM, Mundy C, Banerjee A, Kwanjana JH, Salaniponi FML (2000) Screening tuberculosis suspects using two sputum smears. Int J Tuberc Lung Dis; 4 (1): 36-40.

[8] Ozkutuk A, Terek G, Coban H, Esen N (2007). Is it valuable to examine more than one sputum smear per patient for the diagnosis of pulmonary tuberculosis? Jpn J Infect Dis; 60: 73-75.

[9] Yasin MA, Cuevas LE (2003). How many sputum smears are necessary for case finding in pulmonary tuberculosis? Tropical Medicine and International Health; 8 (10): 927-932.

[10] Gopi PG, Subramani R, Selvakumar N, Santha T, Eusuff SI, Narayanan PR (2004). Smear examination of two specimens for diagnosis of pulmonary tuberculosis in Tiruvallur District, South India. Int J Tuberc Lung Dis; 8 (7): 824-828.

[11] Sarin R, Mukerjee S, Singla N, Sharma PP (2001). Diagnosis of tuberculosis under RNTCP examination of two or three sputum specimens. Ind J Tub; 48: 13-16.

[12] Thomas A, Chandrasekaran V, Joseph P, Rao VB, Patil AB et al (2008). Increased yield of smear positive pulmonary TB cases by screening patients with $>2$ weeks cough, compared to $>3$ weeks and adequacy of 2 sputum smear examinations for diagnosis. Indian J Tuberc; 55: 7783. 